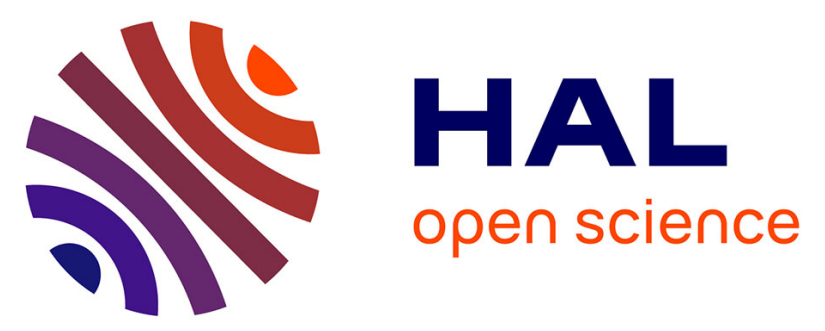

\title{
RAPP: A Robotic-Oriented Ecosystem for Delivering Smart User Empowering Applications for Older People
}

Sofia E Reppou, Emmanouil G. Tsardoulias, Athanassios M Kintsakis, Andreas L Symeonidis, Pericles A Mitkas, Fotis E Psomopoulos, George T Karagiannis, Cezary Zelienski, Vincent Prunet, Jean-Pierre Merlet, et al.

\section{To cite this version:}

Sofia E Reppou, Emmanouil G. Tsardoulias, Athanassios M Kintsakis, Andreas L Symeonidis, Pericles A Mitkas, et al.. RAPP: A Robotic-Oriented Ecosystem for Delivering Smart User Empowering Applications for Older People. International Journal of Social Robotics, 2016, 10.1007/s12369-0160361-z . hal-01336250

\author{
HAL Id: hal-01336250 \\ https://hal.inria.fr/hal-01336250
}

Submitted on 22 Jun 2016

HAL is a multi-disciplinary open access archive for the deposit and dissemination of scientific research documents, whether they are published or not. The documents may come from teaching and research institutions in France or abroad, or from public or private research centers.
L'archive ouverte pluridisciplinaire HAL, est destinée au dépôt et à la diffusion de documents scientifiques de niveau recherche, publiés ou non, émanant des établissements d'enseignement et de recherche français ou étrangers, des laboratoires publics ou privés. 


\title{
RAPP: A Robotic-Oriented Ecosystem for Delivering Smart User Empowering Applications for Older People
}

\author{
Sofia E. Reppou ${ }^{1}$ - Emmanouil G. Tsardoulias ${ }^{2}$ - Athanassios M. Kintsakis ${ }^{3}$. \\ Andreas L. Symeonidis ${ }^{2}$ - Pericles A. Mitkas ${ }^{3}$ - Fotis E. Psomopoulos ${ }^{2}$. \\ George T. Karagiannis ${ }^{1}$. Cezary Zielinski ${ }^{4}$. Vincent Prunet $^{5}$. \\ ${\text { Jean-Pierre } \text { Merlet }^{5} \text { • Miren Iturburu }}^{6}$ - Alexandros Gkiokas ${ }^{7}$
}

Accepted: 8 June 2016

(C) Springer Science+Business Media Dordrecht 2016

\begin{abstract}
It is a general truth that increase of age is associated with a level of mental and physical decline but unfortunately the former are often accompanied by social exclusion leading to marginalization and eventually further acceleration of the aging process. A new approach in alleviating the social exclusion of older people involves the use of assistive robots. As robots rapidly invade everyday life, the need of new software paradigms in order to address the user's unique needs becomes critical. In this paper we present a novel architectural design, the RAPP [a software platform to deliver smart, user empowering robotic applications (RApps)] framework that attempts to address this issue. The proposed framework has been designed in a cloud-based approach, integrating robotic devices and their respective applications. We aim to facilitate seamless development of RApps compatible with a wide range of supported robots and available to the public through a unified online store.
\end{abstract}

Sofia E. Reppou

sreppou@gmail.com

1 Ormylia Foundation, Diagnostic Center, Ormylia, Chalkidiki, Greece

2 ITI-Information Technologies Institute, CERTH-Centre for Research and Technology Hellas, Thermi, 57001 Thessaloníki, Greece

3 Department of Electrical and Computer Engineering, Aristotle University of Thessaloniki, 54124 Thessaloníki, Greece

4 Institute of Control and Computation Engineering, Warsaw University of Technology, ul. Nowowiejska 15/19, 00-665 Warsaw, Poland

5 Inria, Sophia-Antipolis, France

6 Matia Instituto Gerontologico, Donostia-San Sebastian, Spain

7 Ortelio Ltd., Coventry CV1 2TT, UK
Keywords Robotic applications · Cloud robotics · Robotic architecture - Mild cognitive impairment - Assistance robotics $\cdot$ Health monitoring $\cdot$ Social robotics

\section{Introduction}

It is becoming increasingly evident that our current social infrastructures and services are struggling to keep up with the dramatic demographic changes apparent in our societies. As depicted in Fig. 1 the share of people aged 65 years or over in the total population in Europe (EU28) is projected to increase from $18.5 \%$ in 2014 to $28.4 \%$ in 2060 and the respective absolute number is projected to rise from 93.9 to 148.4 million in 2060. It is a general truth that in the near future, older people requiring support in their daily life will increase dramatically, overburdening the available caregivers [27]. Socially interactive robots can prove helpful, not only by physically assisting people, but also by monitoring their health state and ensuring their safety while also functioning as a companion [4]. The increasing sales figures of robots are indicating that we are witnessing a rising trend for social robotics $[14,20,32,37]$. In order to lower the cost for developers and to increase their interest on developing robotic applications (RApps), the RAPP project introduces the idea of robots as platforms [28].

The RAPP project, aptly named "Robotic Applications for Delivering Smart User Empowering Applications", aims to provide an open-source software platform to support the creation and delivery of RApps. A common application programming interface (API) provides developers the needed functionalities for implementing RApps, as well as accessing the robot's sensors and actuators using higher level commands. This is achieved by inserting a middleware stack with added functionalities suitable for different kinds of robots. 
Fig. 1 Population pyramid for EU27, 2008 versus 2060

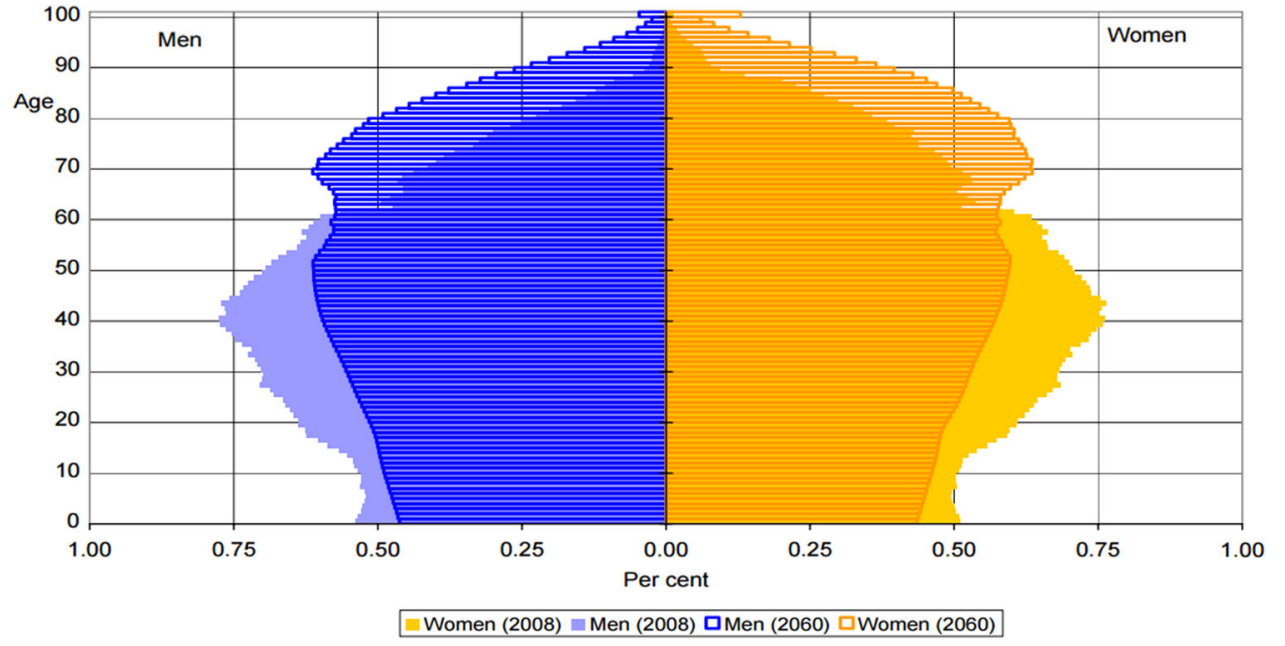

RAPP also expands the computational and storage capabilities of robots and enables machine learning operations, distributed data collection and processing, and knowledge sharing among robots in order to provide personalized applications adapted to the individual. The use of a common API assists developers in creating improved applications for different types of robots, whilst addressing people with different needs, capabilities and expectations, and at the same time respecting their privacy and autonomy. The RApps will be hosted in an online store, the RAPP Store, which is expected to ultimately have a profound effect in the RApp market.

RAPP aspires to foster the development of robots and RApps to encourage social, emotional, and cognitive empowerment of people at risk of exclusion, and in particular older people, including those with social or cognitive deficits. Developing policies to confront and prevent the social exclusion of older people is a major concern of the World Health Organization and all European countries [43].

The need for this technology is driven by critical societal problems requiring sustained, personalized support, supplementing the efforts of caregivers, social workers, clinicians and relatives attending older people. The RAPP project will take into consideration the real needs and expectations of older people, and provide a framework of high motivation and trust for older people. The proposed technologies will be integrated, taking into consideration the importance of the surrounding social environment. RAPP will offer solutions that help older people remain socially active, increasing their independence and autonomy, while relieving helpers from tedious tasks and providing tools for the medical community for a better assessment of the functional and cognitive status of the subject.

The rest of the paper is organized as follows. Section 2 describes the targeted user groups. Section 3 presents an overview of the RAPP technical architecture and the services it provides to the targeted user groups. Section 4 introduces the robots we are currently using in RAPP and presents the pilot scenarios in detail, as well as some early results. Section 5 mentions our future work plans and finally Sect. 6 concludes the paper.

\section{RAPP Target Users}

The initial target group of the RAPP project are older people in high risk of social exclusion and specifically those that either (a) are technology illiterate, (b) were diagnosed with mild cognitive impairment (MCI), or (c) suffer from mobility related disabilities.

\subsection{Technology Illiterate People}

No one can deny that technology is an integral part of life in the twenty first century. When it comes to performing simple money transactions, communicating with other people via phone or email and searching for information on the internet, all are performed through interaction with digital devices, namely computers and mobile devices. As a result, everyone is required to be technologically literate in order to understand technology, be able to use technological devices and processes and participate in society's decisions on 'technological' issues [7]. Nowadays, all people should be able to understand, evaluate, use and adapt to emerging technological innovations [1]. We must not forget though that part of the population may have difficulties gaining access to these innovations or reaching a high level of computer literacy.

As people get older, a number of age-related changes happen that make interaction with technology even more challenging: their vision and hearing declines, their motor skills decrease and their cognitive skills deteriorate. Their social life may also suffer as health issues and disabilities restrict them inside the house, leading gradually to social 
exclusion. Technology in general and computers in particular may provide a partial answer to the social exclusion of older people by helping them focus on social relationships by relieving them from tedious, physically demanding tasks and facilitating social contact, decreasing isolation, assisting in learning opportunities and supporting older people in acquiring new skills. The aforementioned experiences cultivate a feeling of self-esteem, joy, accomplishment and a sense of connection within the modern world. Unfortunately, as older people feel alienated to computer technology, the process of familiarizing themselves is filled with feelings of anxiety and reluctance.

RAPP is challenging the barriers and the common conception that older people are unwilling and unable to adopt modern technologies and applications like email, search engines or social networks. RAPP will present applications that will enable older people to indulge in technology under the guidance of their assistant robot while providing useful help for the helpers and assessment tools for the medical community.

\subsection{People with Mild Cognitive Impairment}

As we grow older, a lot of physical changes happen to our body. Numerous physiological, functional and neurocognitive changes, subject to a high level of variance across the population, affect the brain. The risk of dementia increases affecting the daily life of seniors. The prevalence of dementia in European countries is about $6.4 \%$ in people over 65 years old, and this percentage doubles every 5 years after this age $[25,35]$. The number of people living with dementia worldwide is currently estimated at 47.5 million and is projected to increase to 75.6 million by 2030 . The number of cases of dementia are estimated to more than triple by 2050 [44].

The first stage of dementia is characterized by a subtle but noticeable memory loss, presenting as a tendency to forget recent information. This stage, described as an intermediate state between the expected cognitive decline of normal aging and the more serious decline of dementia [18], is defined by the term of MCI. Apart from memory problems, people suffering from MCI can also present difficulties with language, reasoning and judgement that are not justified by their age [15]. Their cognitive dysfunction may not significantly impair their daily life activities but, being aware of the process, it causes them feeling of anxiety and frustration. Furthermore, it is noticeable by others (family and friends) and can be measured in clinical assessments [6]. MCI gradually progresses to full blown dementia and Alzheimer but early and appropriate therapeutic interventions might delay progression and maintain this stage for a long time or, in some cases, even return to normal cognitive function for their age [21].
World Health Organization recognizes dementia as a public health priority [44]. Unfortunately, there is no treatment to cure or slow down the progressive course of dementia although numerous symptomatic treatments can improve the quality of life of patients [31]. Drugs that are used to treat symptoms of Alzheimer's disease were not shown to benefit MCI patients by either delaying or preventing progression to the next stage of dementia [2]. According to Tiberio et al. [40] people with MCI can benefit from cognitive training, both computer-based and non, as this way they maintain the cognitive ability to learn new information and use memorization tactics.

Robots can provide an innovative solution to multiple issues that people with MCI are facing, including but not limited to, helping them with their medicine schedule, dates and daily tasks; enhancing their social life and social interaction by facilitating their communication with family and friends through internet applications and preventing them from feeling socially isolated or lonely by promoting a socially and mentally stimulating companionship. The initial applications of the RAPP project are oriented towards this direction.

\subsection{People Requiring Mobility Assistance and Activity Monitoring}

Risk of fall and mobility problems are two of the most common and serious concerns that older people are facing, as even a relatively mild fall may lead to fractures, soft tissue injuries and even death [13]. Bone density diseases like osteoporosis and osteopenia, which primarily target older people, drastically increase the risk of fracture accompanied to a fall or injury. Hip fractures are one of the most serious fall outcomes among older people, being associated with high morbidity, mortality, disability and subsequent hospital and societal costs as well as a reduction in quality of life [12].

Worldwide, 1.6 million patients suffer from a hip fracture each year and as the population continues to age this figure is projected to increase by $25 \%$ each decade. A hip fracture has devastating consequences in older people [29]. Hip fractures often affect the functioning and autonomy of older people [17], and are associated with an overall mortality of 22-29\% one year after the injury [17]. Furthermore, $27 \%$ of older people require a walking aid 1 year after a hip fracture surgery [16].

The goals of a device assistive to the rehabilitation process are to improve independent mobility, reduce disability, delay functional decline, decrease the burden of care and provide assessment tools for the medical community [3]. Patients using assistive devices have reported improved confidence and feeling of safety resulting in increased activity levels and independence [38]. Assistive devices such as canes, crutches, and walkers can be used to increase a patients base of sup- 
port, improve balance and increase activity and independence without interfering with the rehabilitation outcomes [26].

RAPP will apply the most innovative solutions to provide support for people with mobility problems and who are under a rehabilitation process. RAPP will identify and personalize the provided support according to each user's features and needs while also motivating them to improve their activity level and perform exercises adapted to their physical status. Moreover, RAPP will monitor the walking parameters of the patient in order to analyze and report his walking pattern and any subsequent improvements to his professional therapist.

\section{RAPP Architecture}

In order for the reader to familiarize himself with the RAPP project, a brief description of the RAPP architecture depicted in Fig. 2 follows [33]. RAPP is designed as with a distributed architecture in mind and consists of two major layers: the RAPP platform (located on the cloud) and the robot platform (located on each robot supported by RAPP). Within each layer, subcomponents exist and are organized into entities called agents, namely being the dynamic, platform, cloud and core agents. RApps utilize at a minimum the dynamic agent and one or more of the other agents.

The RAPP platform comprises the following six major components.

- The RAPP Store where a collection of RApps that users may download on their robots is hosted.

- The RAPP Improvement Centre, otherwise denoted as platform agent, which is responsible for providing a number of cloud-based services including machine learning support, multimedia enhancement and robotic algorithms.

- The knowledge base which is an ontology based on the KnowRob [39] robotic ontology and further enhanced with social concepts [41]. It serves as a central knowledge management system for all the robots participating in the RAPP ecosystem.

- The centralized MySQL database which is the central data repository of the RAPP platform.

- The offline learning processes is a collection of machine learning components that periodically analyze data originating from within the knowledge base and the centralized database in an attempt to extract meaningful knowledge.

- The RApp execution workspace, otherwise denoted as the cloud agent, providing developers the functionality of a common workspace to utilize when desiring to offload part of their custom application directly to the cloud.

On the other hand, the core agent, located on the robot side, provides the means for the robot to connect and interact with the RAPP Store. Furthermore, the core agent is responsible for providing an abstraction layer for the low level robotic services responsible for retrieving readings from the robots sensors and task commands to its actuators. The dynamic agent is the actual RApp being executed within the robot. It can utilize the provided platform and core agent services and optionally make use of the cloud agent. The communication layer acts as the bridge between the core and the dynamic agent.

\subsection{Data Privacy and Security}

In order to address data privacy and security concerns the RAPP system was designed according to the latest data transmission and security protocols upon which similar systems are based and are already in widespread use. More specifically:

All communications to and from the RAPP platform and the robot are authenticated and data transmissions take place through the secure and encrypted https protocol. All RAPP project specific user data is stored in the ontology knowledge management system anonymously, meaning that only a random string of characters and numbers, denoted as the user's ontology alias, ties the actual user to his data. No sensitive information is stored like names, addresses, usage logs or multimedia files that can be tied back to a specific user directly.

A link does exist between a user's name and personal information and his ontology alias, but that link is stored in a secure MySQL database which is separate of the ontology knowledge management system and most importantly the database is inaccessible from the outside world. Specifically, we explicitly deny access to the database from RApps, thus even in the case that a developer or user decides to perform malicious activities they will have no access to it. Access to the database can only be obtained by the services running on the RAPP platform which have been developed as part of the RAPP system architecture and are shielded against malicious attempts to hack the database (SQL injections) according to the latest and established software practices.

RApps are running in sandboxed mode on the robot side, making it impossible to intervene with the execution of other RApps running simultaneously. Even if that was the case though, as no RApp has access to personalized data, it would be impossible to obtain such information by tampering with the execution of an active RApp.

The whole RAPP platform and store are running on a private cloud infrastructure used solely for the purposes of the RAPP project. No other application or system is running on the same infrastructure and no other user, entity or company not involved in the RAPP project can access it. The only way to access sensitive user data is to have physical access to the infrastructure where RAPP is hosted. 


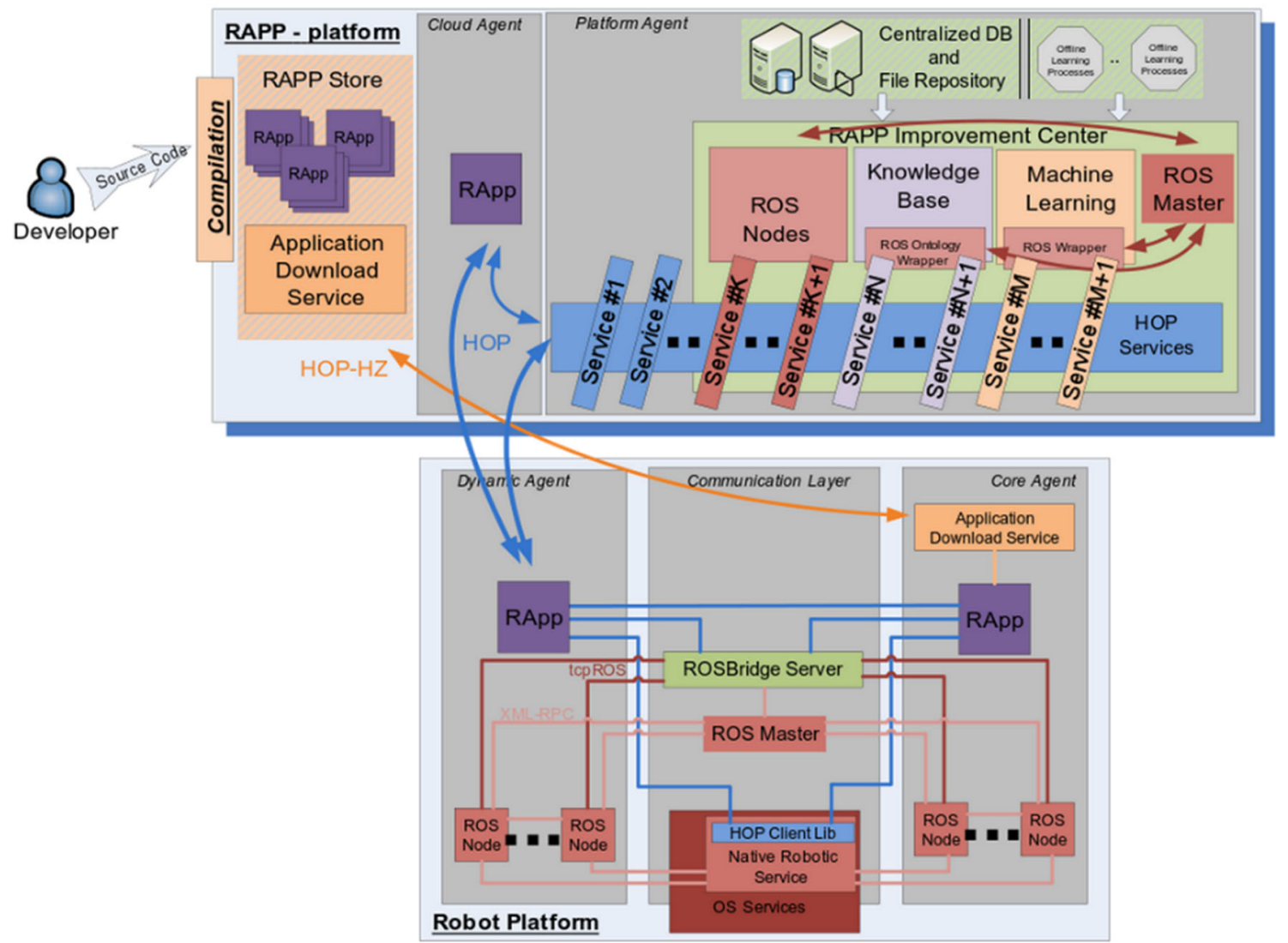

Fig. 2 Overview of the RAPP architecture

As it is theoretically possible for a malicious developer to create a RApp that undertakes behavior undesired by the user (monitoring him, for example) without his knowledge we plan to review all RApps submitted to the RAPP store in order to prevent such action, when the RAPP store reaches a production state. However, this issue is not specific to the RAPP project as theoretically any application currently existing in online smartphone application stores could undertake such behavior. The only mechanism to ensure proper application activity is analyzing its source code, and this is the approach followed by all major application stores.

Before downloading and using a RAPP, the user will be notified whether it has been reviewed and deemed safe or not and will be prompted to act accordingly. It is our opinion that older people and especially those with some form of cognitive impairment should be prevented from using RApps that have not been deemed safe and appropriate, thus a special lock mechanism exists that will prevent them from downloading and using them.

We are confident with the data privacy and security measures in place in order to prevent unauthorized access to sensitive user data. Of course, limitations may exist in our approach, but are equivalent to those of all online public services in our society. Regarding privacy issues, we can guarantee that the user data we accumulate will not be used for purposes not stated in our agreement with the user and beyond the scope of goals of the RAPP project.

\subsection{RAPP Platform Services}

The RAPP platform provides a number of services offering high level functionalities that are critical to the function of most RApps. These services efficiently address a number of problems that require a significant level of expertise due to the actual algorithms and methods involved. As such, we chose to implement state of the art solutions and package them in a user friendly approach that RApp developers can easily use to enhance their applications by invoking off-the-shelf web services. The following subsections summarize the RAPP platform services that were used in the RAPP pilot cases and scenarios.

\subsubsection{RAPP Ontology}

The RAPP ontology serves as a central repository and knowledge management system for all robots participating in the RAPP ecosystem. The main concept of an ontology is that its classes and attributes are semantically linked according to 
the principles of inheritance and as such new knowledge can be derived either directly or indirectly. Our ontology scheme is based on the KnowRob robotic ontology.

In our implementation, the pure KnowRob ontology was enhanced after the insertion of extra classes derived from the OpenAAL ontology, as well as others needed to implement the desired RApps. KnowRob is not just the OWL file containing the ontology, but a complete framework that provides an abundance of functionalities and interfaces. It is useful to state that the core of KnowRob was altered in order to perform checks and allow for extra functionalities. Higher level Prolog functions were included that address common ontology use cases like creating instances and assigning attributes and submitting complicated queries.

As the knowledge base expands, new robots will be instantly connected to the collective intelligence of the RAPP ecosystem. By effortlessly tapping into existing knowledge, new robots can reach the top of their learning curve, thus achieving an exponential overall learning rate.

\subsubsection{RAPP Cognitive Exercise}

The RAPP cognitive exercise system aims to provide the robot users a means of performing basic cognitive exercises [22]. The tests are offered as services residing in the platform agent. The cognitive tests supported belong to three distinct categories, (a) arithmetic, (b) reasoning/recall, (c) awareness. A number of subcategories exist within each category. Tests have been implemented for all subcategories in different variations and difficulty settings. A household robot equipped with speakers and a microphone with recording capabilities is used in order to dictate the test questions to the user and record the answers.

A user performance history is being kept in the ontology that aids in keeping track of the users cognitive test performance and in adjusting the difficulty of the tests he is presented with. Based on past performance the test difficulty adapts to the users specific needs in each test category separately in order to accurately reflect the users individual cognitive strengths and weaknesses. To preserve the users interest different tests are selected for each category using a least recently used model. To further enhance variation, even tests of the same subcategory exist in different variations.

\subsubsection{RAPP Speech to Text/Text to Speech}

In our case we desire a flexible speech recognition module, capable of multi-language extension and good performance on limited or generalized language models. For this reason, Sphinx-4 [42] was selected. Since we aim for speech detection services for RApps regardless of the actual robot at hand, we decided to install Sphinx-4 in the RAPP cloud and provide services for uploading or streaming audio, as well as configuring the ASR towards language or vocabulary-specific decisions.

The configuration capabilities of Sphinx- 4 contain an acoustic model, a language model and a phonetic dictionary. In addition, Sphinx-4 also possess the capability to employ a grammar imposing sets of rules that provide a more limited but highly accurate word recognition, that can be ideal for command and control scenarios.

Speech to text capabilities are provided to robots that are equipped with microphones but do not inherently support by a RAPP platform cloud service utilizing the eSpeak speech synthesis library [9], as well as the MBROLA [8] project that specializes in altering speech synthesis voices.

\subsubsection{RAPP Face Detection}

The RAPP face detection service tries to detect faces in arbitrary images according to the latest trends in the image manipulation scientific area. It should be stated that the face detection problem is different from the face recognition, as the first tries to detect if some faces exist in a given frame, whereas the second must identify the person this face belongs to. In the RAPP ecosystem, such functionality is of utmost importance as we desire for robots to actively interact with elder users. Thus the actual utilization may be multiple:

- Human detection in the house for interaction or tracking purposes,

- User recognition for personalization purposes,

- Face tracking for mimicking human motions when interacting with the elders,

- Emotions recognition by facial characteristics.

Obviously, in order for a robot to perform face detection, it must be equipped with a camera sensor, having the necessary field of view and resolution. In RAPP, face detection will be utilized by household robots that are equipped with cameras. It should be stated that for real-time applications, the face detection algorithm is able to lower the resolution of the image in order to achieve processing at a higher frame rate per second.

\subsubsection{RAPP Image Recognition}

The RAPP image recognition service utilizes Caffe, an open source framework [19] for state-of-the-art deep learning algorithms along with a collection of reference models, developed and maintained by the Berkeley Vision and Learning Center (BVLC) and an active community of contributors on its GitHub repositories. The main application of Caffe is the recognition of objects within an image. 
For our use case we have adopted the BVLC reference pre-trained model for image recognition which is capable of identifying a substantial number of objects found within a household environment. The image recognition service is often used in collaboration with the ontology knowledge management service in order to extract meaningful knowledge following the identification of an object. This is particularly useful in scenarios involving robot interaction with objects where following the identification of an object, a series of actions is to take place. The hazard detection is such a use case scenario and one of the first RApps we have developed. In this scenario, the robot is tasked with taking images around the house, identifying potential objects or situations as hazardous (for example an open door, or window at night) and notifying the senior to proceed with corrective action.

\subsubsection{Audio Processing}

The audio processing service was created in order to perform the necessary operations for the speech recognition modules to operate for all audio cases. The RAPP speech detection services can operate either with ogg or with wav (one and four channels) by employing the audio processing node. The audio processing service is tasked with converting the audio source single channel wav files, with a $16 \mathrm{kHz}$ sample rate and 16 bit little-endian format as Sphinx-4 requires. As is often the case, the robot captured audio can be noisy or distorted and as such produce undesired output when directed to the Sphinx-4 speech recognition service.

It is obvious that in order for the speech recognition modules to operate successfully, denoising operations must take place. Additionally, since each robot creates recordings with noise patters of different spectral characteristics, a personalization effort must be performed, storing silence samples from each robot and extracting the noises DFT coefficients. These denoising operations are offered as services by the audio processing package. This node utilizes the SoX Unix audio library in order to perform spectral denoising, along with other custom made techniques.

\subsubsection{RAPP News Explorer}

The RAPP news explorer service enables RApps to search news on websites/engines (i.e., Google News). Access to newspapers and magazines could be difficult for older people due to vision deficiencies or motor disabilities. This RAPP service is tasked with streaming news content according to predefined user topics of interest in order for older adults to be entertained, informed and up to date. Depending on the actual robot, content can be delivered to the user either as an audio file which the robot will pronounce or be read directly as text from a screen in case the robot possesses one.

\subsubsection{RAPP Weather Reporter}

Older people are particularly interested in weather forecasts due to the weather being a critical factor affecting their health issues and leisure activities. This RAPP service is tasked with retrieving weather forecasting data for the location of the user. Again, depending on the actual robot, content is delivered to the user either as audio pronounced to the user, or directly to the robot's screen. The service also supports summarizing the weather report and displaying personalized warnings to the user, for example warning people with arthritis about high humidity or air pressure changes.

\subsubsection{Mail Management}

The mail management service is responsible for being the proxy between the end user and the mail provider. As one of the RAPPs pilot cases concerns the technology illiterate, this node will provide an easy and effortless way for older people to actively use (send and receive) mails. The node will utilize the SMTP server infrastructure installed on the RAPP platform and will provide two authenticated services: one for sending mails and one for fetching. The email related credentials information is stored in the MySQL database.

\subsubsection{QR Detection}

QR is a kind of barcode, with the exception that common barcodes are one dimensional, whereas QRs are two dimensional, thus able to contain much more information. A QR consists of square black and white patterns, arranged in a grid in the plane, which can be detected by a robot's camera in order to perform the decoding process. A special service has been developed that acts as a wrapper to the well-known ZBar library, in conjunction to OpenCV for image manipulation, in order to read QR codes. QR codes find significant application in the RAPP project as they can be employed in a variety of ways, including visual tagging of objects for easier object detection, or even tagging of semantic spatial information, like rooms, furniture, etc., that will help in the mapping, localization and navigation procedures of the robot.

\section{The RAPP Robots and Scenarios}

RAPP's initial objective is to include two robotic platforms into the RAPP development cycle: the Aldebaran NAO humanoid ${ }^{1}$ robot, and INRIA's ANG-MED ${ }^{2}$ robot. The reason behind the use of two rather different robots should be

\footnotetext{
${ }_{1}$ http://www.aldebaran.com/en/humanoid-robot/nao-robot.

2 https://pal.inria.fr/research/themes/rehabilitation-transfer-and-assis tance-in-walking/walking-aids/.
} 
obvious. We need to demonstrate platform interoperability and to provide evidence of the generalization of the RAPP concept by wrapping different capabilities through the RAPP API. In addition, it has not yet been resolved as to whether humanoid or custom robotic platforms are better suited for robotic aid's needs, thus we have to study both approaches and shed some light in either direction. The RAPP applications (RApps) are going to be tested in three distinct pilot cases, each targeting a different user group.

\subsection{Technology Illiterate Group}

Seniors citizens from the local Community Centre at New Moudania (Greece) participated in the pilot testing RAPP scenario of technology illiterate people interacting with a robot, NAO in this case [34]. Their computer knowledge was non-existent or minimum; they didn't know how to use email, Skype or social media. Our hypothesis was that NAO could improve their social life by connecting them with friends and family faster and easier than learning how to use computers. We aspired to demonstrate how easily NAO could accomplish this connection when conventional computer training is prohibited due to the time and effort involved. Moreover, we would like to examine if NAO, as a companion robot, could improve the psychological status of older people and assert a positive effect on their mood and emotions. In order to test our hypothesis, we assembled a group of 18 elders to participate in our research. The members of the group have already started to attend computer and technology related classes and familiarizing themselves with the robot NAO and its functionalities.

To establish the integrity of the pilot test case, we decided to use a focus subgroup of 6 people, selected from our group of 18 older people comprising mostly of those who are frequently attending our meetings. Another group of six participants was chosen among the older people of the Community Seniors Centre to serve as the control group.

\subsection{Mild Cognitive Impairment Group}

Seniors diagnosed with MCI will participate in this pilot case by using and testing applications that support user personalized cognitive exercising. These applications aim to enhance the attention, reasoning and recall capabilities of the patients while simultaneously keeping track of their performance in order to assist their caregivers in evaluating their progress and adjusting the exercise program accordingly. The exercises will resemble games and NAO is going to interact with individuals in a playful mode in an attempt to make the experience enjoyable. Both cognitive and emotional outcomes will be evaluated as we expect that NAO will be more effective than simple written or computer exercises, causing less stress while promoting enjoyment and satisfaction. In addi- tion, performing cognitive exercises with NAO's assistance does not necessitate the involvement of special trainers or any computer knowledge making it ideal to incorporate in a daily basis while simultaneously keeping subsequent costs at a minimum.

\subsection{Hip Fracture Rehabilitation group}

The ANG-MED robot, a stable and robust smart rehabilitation walker that can be programmed to take into account the user's specific needs is being evaluated in this pilot case by a group of older people with hip fracture in San Sebastian (Spain). The ANG-MED is able to identify the user, download his profile and adjust its settings accordingly. Moreover, it is able to detect the correct position of the user and assist him in adjusting it if improper. The ANG-MED robot promotes a feeling of safety as it holds the user stable and allows him to take a few steps without fear of falling and even if this would happen the rollator would alarm the caregivers in time to prevent an accident. Furthermore, it assists users in performing specific exercises to train their muscles and speed the recovery and rehabilitation process. It also provides proper guidance to the users in order for them to correctly perform their exercises. Caregivers can monitor the performance of the users through the data collected by the ANG-MED robot and are able to evaluate their rehabilitation and adjust the exercises to their needs.

\subsection{Early Results}

Although the development of RAPP applications has not yet completed, we decided to proceed with early tests of the initial applications to examine their robustness and usability and collect feedback from the users. Observing the human-robot interaction and discussing with users their experience gave us an interesting feedback to improve or adjust the offered applications addressing the real needs of the users. The applications already implemented and ready for testing were the email handler and the cognitive games, both tested with the NAO robot, as well as the dynamic and static exercises tested with the ANG-MED robot.

For evaluating the performance of the mobility assistance pilot case, already existing medical tests have been used in order to use the same indicators that have been medically validated. There are also additional indicators that have been medically established but will become standards only when sufficient statistical data have been obtained (this may take years) and devices that make them measurable are commercially available. As will become apparent, the tests undertaken have demonstrated the viability of the RAPP project software architecture. A web user interface is available for caregivers and patients in order to provide feedback on the applications. 


\subsubsection{Cognitive Games and Email Handler Pilot Cases}

The cognitive games application offers a number of cognitive exercises to users who either require or wish to enhance their attention and improve their memory skills. The offered exercises consist of:

(1) Arithmetic calculations simple-to-complex arithmetic quizzes

- Basic arithmetic tests "How much four plus 3 equals?"

- Money-change tests "We buy a carton of milk which costs one euro and twenty cents and we pay with a two euro coin. How much change do we get back?"

- Time difference tests "You have an appointment with the doctor at, fifteen minutes past seven in the evening. You reach his office by seven and the doctor takes you in after twenty five minutes. At what time did the doctor take you in?"

(2) Awareness exercises

- Quizzes related to everyday perception "What day/ month/year is today?"

(3) Reasoning short story-telling and memory related quizzes

- Story-telling tests short stories and a set of questions and predefined answers.

- Word-remembering tests "I am going to say a few words; and then I am going to ask you to repeat them in the same order..."

All the above exercises are provided in varying grades of difficulty and users can start from the easiest level and move on to more difficult ones according to their performance. The email handler enables the user to send emails and attach image and sound/audio files, receive emails, be alerted about incoming emails and listening to them through text to speech procedures.

For the needs of the pilot testing of the email handler and cognitive games applications with NAO, the focus group of the technology illiterate seniors $(\mathrm{N}=6)$ and a group of professionals working with older adults diagnosed with MCI $(\mathrm{N}=10,6$ psychologists, 2 physiotherapists, 1 social worker, 1 gymnast specialized in physical training for older adults) was used. We have chosen at this initial point to work with small focus groups as this gave us the opportunity to discuss in detail and analyze their experience with the robot and the performed applications and collect their suggestions for improvements. Due to the small number of users, our approach was focused on observations regarding the human-robot interaction and performing personal and group interviews of the users. Furthermore, we have noticed that questionnaires are not welcomed by older adults as they were tiring to complete or confused them by the Likert scale and tended to complete them by giving random answers or copying the answers of other users near them.

The focus group of older adults $(\mathrm{N}=6)$ tested NAO by performing the email handler scenario first and the cognitive game scenario second. At the end of testing, a personal interview followed where the participants were asked about their experience with NAO and their evaluation of the applications and the robot's performance. A short questionnaire with open ended questions was used to structure the interview. The participants were asked to describe:

- The general performance of NAO in a scale from 1 to 5 where 1 stood for not satisfied at all and 5 for very satisfied,

- What might have been difficult for them,

- What they would like to change at the procedure,

- If they would rather sent emails through personal computers or through the robot,

- If they would like to exercise their memory with cognitive games like the one presented to them during the tests,

- If they would rather exercise with cognitive games through personal computers or through the robot,

- If they would like to leave a comment.

The interviews revealed that they approved the procedure in both RApps and found them interesting, useful and easy to use. Concerning the usability of RApps, four out of six users (4 in 6) commented that they would rather use RApps and robots while one ( 1 in 6 ) stated that would use both robots and computers for sending emails and play cognitive games. In order to explore in detail the feelings of the users towards robots, we used the negative attitudes towards robots scale by Nomura to investigate potential negative perceptions and behaviors that could prevent interaction between robots and older people [30]. The questions involved were posed orally rather than in a distributed questionnaire (for the aforementioned reasons of small sample and difficulty with questionnaires) and were analysed and discussed in detail.

The main outcome of this open discussion was that older people imagine robots in a human-like form moving around the house performing household chores and taking care of them like "mechanical servants". They would like robots to have feelings and make friends with them but they do not really believe that this could happen, at least not in the immediate future. When insisting on this aspect (imagine that we could have a robot with feelings by tomorrow), they expressed some concern about how the world would be if people made friends with robots instead of each other or how complicated the human-robot interaction would be if feelings were engaged. The basic conclusion was that robots are useful as assistive machines and there is no reason to 
worry as they will always be like that; all the scenarios about them having feelings or thinking and acting independently are impossible (this is sci-fi).

The group of professionals $(\mathrm{N}=10)$ tested the same RApps of email handler and cognitive games and provided feedback on the usability of the applications. They pointed out that applications should be structured to:

- motivate users to utilise them,

- have simple steps to complete a task

- do not have procedures that need to be memorised (passwords, menu paths),

- have clear messages for successful managing of the procedure,

- be adjusted to the personal needs of the users,

- be personalised (identify the users and adjust to their preferences),

- have an adequate sound system. The robotic voice should be clear, nice and easy to understand. The sources of noise from the system should be diminished so that the hearing of the robot should be sufficient and the users will not be obliged to repeat their commands or answers.

Concerning the interaction with the robot, they perceived NAO as:

- friendly and nice to interact with,

- cute and resembling to a kid, not scary at all,

- they would like to have him at home

- easy to use and interact with.

As usability can be defined as the interaction between the user and the system and can be evaluated according to the experience of the users, their satisfaction from the product or system and its acceptability [5], initial testing has already shown that RApps are successfully addressing these principles. Observations and interviews (both personal and group) with the initial user group of technology illiterate older adults have revealed that RApps via NAO were pleasurable and satisfying. The users enjoyed to interact with the robot with which felt very familiar after their first meeting. Their performance on using the applications was exceptional as they found them built in simple and easy steps.

Fisk et al. [10] argued that usability issues depend on the design of the product and the provided training. The design of the product should take into account the physical and cognitive changes that occur with ageing and be formed according to the following five traits [11].

- Learnability the time needed to successfully complete a task and the specific time needed to obtain the results.

- Efficiency the satisfaction of the user when using the product.
- Memorability the time needed to perform a task that has been previously experienced.

- Errors the incidence of product errors and the time needed to recover these errors.

- Satisfaction pleasure derived from using the product.

In the case of RApps, all the above traits are successfully addressed as the offered applications are simple to use and therefore easy to be learned and remembered, they are fun and provide satisfaction to the users, software errors occurred up to now were minimal and were promptly addressed.

Finally, as RAPP is targeting social inclusion and NAO is a companion robot, the provided applications (RApps) were positively evaluated by users as enhanced activities of daily living (EADL) according to Rogers et al. [36]. EADLs are activities related to the quality of users life, engaging them in educational opportunities and cognitive training, enhancing their social life by facilitating their communication with family and friends and providing leisure activities [23,24].

\subsubsection{Dynamic and Static Rehabilitative Exercises Pilot Case}

The ANG-MED robot was tested at lab environment in Matias facilities by researchers of the Matia Institute $(N=6)$ and care professionals $(\mathrm{N}=10)$ of the Hospital Bermingham. As part of the informed consent process, the participants were informed about the tests and what their participation consisted of. Additionally they were informed about the protocol followed to ensure the privacy of the collected data was explained and a contact person was provided. Participants had to perform several tasks using the ANG-MED robot. Afterwards they answered a questionnaire to show their opinion and recommendations for the improvement of ANG-MED.

Participants were divided into two groups, one group performed the tasks using the rollator in the role of a caregiver $(\mathrm{N}=9)$ supervising the activities performed by older people behind the rollator, while handling the remote control and the users guide; and the other played the role of an older person $(\mathrm{N}=7)$ using the rollator and performing the exercises. Testing was carried out in groups of two-three people. When the group consisted of three people, two of them played the role of the caregiver and one the role of the older person. It should be mentioned here that the care professionals that tested ANG-MED at the lab will be those participating in the pilot at hospital.

The dynamic and static exercises with the ANG-MED robot included: dynamic walk exercises including $10 \mathrm{~m}$ walking, time up and go, inverted $\mathrm{L}$ and maze, as well as static exercises including hip extension, hip abduction, plantar flexion and hip flexion. More than 100 test trials were performed covering the entire aforementioned features and their 
variations. The ability of the smart-rollator to monitor the process and collect the appropriate data was also thoroughly tested.

Regarding patient feedback, 9 participants out of 10 thought that the instructions provided by the users guide are easy to understand and follow. Seven participants out of nine indicated that initializing the robot was easy. However, two participants found it confusing. All participants said that identifying the users by RFID tags was easy. Eight participants out of ten indicated that using the remote control was easy. They recommended some changes to simplify its use and the positioning of the buttons. All participants thought that performing the exercises on the ANG-MED is easy and productive. However, some caregivers voiced concerns about the braking system and the safety of the patients.

Patient feedback has proven invaluable in establishing the value of the ANG-MED robot in the rehabilitative process while also identifying critical areas for improving its usability and accessibility. The next step involves monitoring the activity and the rehabilitation process of the patients at the Matia institution.

\section{Future Work}

We are currently developing more RApps, most of which are targeted towards use cases where the NAO robot is involved. These are:

- Calendar-medication alerts and important dates reminder assisting the user to successfully follow his medication schedule, his appointments with doctors and family anniversaries (birthdays, etc.).

- Hazard detection detection of open doors and windows and alert the user to act accordingly.

- News explorer use search engines to find news or articles.

- Weather reporter and forecast current weather information as well as weather forecast.

As the supporting RAPP platform services already exist for the above functionalities, we are currently developing the actual RApps on the robot side and working on optimal content delivery to the end user.

We plan to test both the above applications as well as the email handler and cognitive games RApps in a large scale environment where groups of healthy older adults, older adults diagnosed with MCI and caregivers will be involved. Apart from the qualitative method analysis which served well the needs of the research up to now, questionnaires will be distributed to users, caregivers and professionals to collect statistical data for the usability of the applications and the human-robot interaction.

\section{Conclusion}

The RAPP project aims to offer RApps that will assist older adults in their daily life activities such as communicating with friends and family, being informed and up to date with regional and international news, getting updates on the weather conditions and weather forecasts, ensuring their safety with hazard detection, practicing their cognitive skills with games that exercise attention and memory and supporting their rehabilitation after hip fracture with physical exercises.

Apart from the usability and usefulness of the above applications a number of issues arise considering the human-robot interaction and the feelings of acceptance of robots by older people. The feelings evoked in older people when interacting with robots is the key issue we need to clarify in order to promote successful assistive technologies and suitable robotic products. In the following testing period of RApps, the usability and human-robot interaction are going to be examined and analysed in depth; results will be published via the RAPP website and in journal articles.

The results of the RAPP project will have an important impact in many domains. First of all, the project being user driven and relying on real user needs (rather than pushing technology based on implicit assumptions about these needs) brings technology closer to older people who have trouble interacting with it. Most importantly, RAPP will provide developers with the necessary tools and APIs to build their own assistive RApps, in order to improve the quality of life of people at risk of exclusion.

Acknowledgments Parts of this work was supported by the FP7 Collaborative Project RAPP (Grant Agreement No. 610947), funded by the European Commission.

\section{References}

1. Al-Alaoui M, Ohannessian M, Choueiter G, Akl C, Avakian T, AlKamal I, Ferzli R (2008) A pilot project-from illiteracy to computer literacy: teaching and learning using information technology. Int J Emerg Technol Learn 3(3):4-9

2. Allain H, Bentué-Ferrer D, Akwa Y (2007) Treatment of the mild cognitive impairment (MCI). Hum Psychopharmacol Clin Exp 22(4):189-197

3. Bateni H, Maki BE (2005) Assistive devices for balance and mobility: benefits, demands, and adverse consequences. Arch Phys Med Rehabil 86(1):134-145

4. Bemelmans R, Gelderblom GJ, Jonker P, De Witte L (2012) Socially assistive robots in elderly care: a systematic review into effects and effectiveness. J Am Med Dir Assoc 13(2):114-120

5. Bevan N (1995) Usability is quality of use. Adv Hum Factors/Ergon 20:349-354

6. Busse A, Bischkopf J, Riedel-Heller SG, Angermeyer MC (2003) Mild cognitive impairment: prevalence and incidence according to different diagnostic criteria results of the Leipzig longitudinal study of the aged (LEILA75+). Br J Psychiatry 182(5):449-454 
7. Bybee RW (2000) Achieving technological literacy: a national imperative. Technol Teach 60(1):23-28

8. Dutoit T, Pagel V, Pierret N, Bataille F, Van der Vrecken O (1996) The MBROLA project: towards a set of high quality speech synthesizers free of use for non commercial purposes. In: Fourth international conference on spoken language, 1996. ICSLP 96 proceedings, vol 3. IEEE, p 1393-1396

9. Espeak speech synthesis library. http://espeak.sourceforge.net/ index.html. Accessed 22 Jan 2016

10. Fisk A et al (2004) Designing for older adults: principles and creative human factors approaches. Taylor \& Francis, London

11. Fisk AD, Rogers WA, Charness N, Czaja SJ, Sharit J (2009) Designing for older adults: principles and creative human factors approaches. CRC Press, Boca Raton

12. Fried LP, Ferrucci L, Darer J, Williamson JD, Anderson G (2004) Untangling the concepts of disability, frailty, and comorbidity: implications for improved targeting and care. J Gerontol A 59(3):M255-M263

13. Friedman SM, Munoz B, West SK, Rubin GS, Fried LP(2002) Falls and fear of falling: Which comes first? A longitudinal prediction model suggests strategies for primary and secondary prevention. J Am Geriatr Soc 50(8):1329-1335

14. Fujita M (2001) Aibo: toward the era of digital creatures. Int J Robot Res 20(10):781-794

15. Guaita A, Colombo M, Vaccaro R, Fossi S, Vitali SF, Forloni G, Polito L, Davin A, Ferretti VV, Villani S (2013) Brain aging and dementia during the transition from late adulthood to old age: design and methodology of the invece.ab population-based study. BMC Geriatr 13(1):98

16. Guralnik JM, Simonsick EM, Ferrucci L, Glynn RJ, Berkman LF, Blazer DG, Scherr PA, Wallace RB (1994) A short physical performance battery assessing lower extremity function: association with self-reported disability and prediction of mortality and nursing home admission. J Gerontol 49(2):M85-M94

17. Haleem S, Lutchman L, Mayahi R, Grice J, Parker M (2008) Mortality following hip fracture: trends and geographical variations over the last 40 years. Injury 39(10):1157-1163

18. Hamill M, Smith L, Röhricht F (2012) dancing down memory lane: circle dancing as a psychotherapeutic intervention in dementia pilot study. Dementia 11(6):709-724

19. Jia Y, Shelhamer E, Donahue J, Karayev S, Long J, Girshick R, Guadarrama S, Darrell T (2014) Caffe: convolutional architecture for fast feature embedding. arXiv preprint arXiv:1408.5093

20. Kanda T, Hirano T, Eaton D, Ishiguro H (2004) Interactive robots as social partners and peer tutors for children: a field trial. HumComput Interact 19(1):61-84. Retrieved on 8 Nov 2015. http:// citeseerx.ist.psu.edu/

21. Kidd PM (2008) Alzheimer's disease, amnestic mild cognitive impairment, and age-associated memory impairment: current understanding and progress toward integrative prevention. Altern Med Rev 13(2):85

22. Kintsakis AM, Reppou SE, Karagiannis GT, Mitkas PA (2015) Robot-assisted cognitive exercise in mild cognitive impairment patients: the RAPP approach. In: E-health and bioengineering conference (EHB), 2015. IEEE, p 1-4

23. Lawton MP (1990) Aging and performance of home tasks. Hum Factors J Hum Factors Ergon Soc 32(5):527-536

24. Lawton M, Brody EM (1970) Assessment of older people: selfmaintaining and instrumental activities of daily living. Nurs Res 19(3):278

25. Lobo A, Launer L, Fratiglioni L, Andersen K, Di Carlo A, Breteler M, Copeland J, Dartigues J, Jagger C, Martinez-Lage J et al (2000) Prevalence of dementia and major subtypes in Europe: a collaborative study of population-based cohorts. Neurol Minneap 54(11; SUPP/5):S4-S9
26. Lucki K, Bach M (2010) Rollator use and functional outcome of geriatric rehabilitation. J Rehabil Res Dev 47(2):151

27. Lutz W, Sanderson W, Scherbov S (2008) The coming acceleration of global population ageing. Nature 451(7179):716-719

28. Mitkas PA (2015) Assistive robots as future caregivers: the RAPP approach. In: Progress in automation, robotics and measuring techniques. Springer, $\mathrm{p} 171-179$

29. Neto AF, Gallego JA, Rocon E, Pons JL, Ceres R (2010) Extraction of users navigation commands from upper body force interaction in walker assisted gait. Biomed Eng Online 9:37

30. Nomura T, Suzuki T, Kanda T, Kato K (2006) Measurement of anxiety toward robots. In: The 15 th IEEE international symposium on robot and human interactive communication, 2006. ROMAN 2006. IEEE, p 372-377

31. Overshott R, Burns A (2005) Treatment of dementia. J Neurol Neurosurg Psychiatry 76(suppl 5):v53-v59

32. Pollack ME, Brown L, Colbry D, Orosz C, Peintner B, Ramakrishnan S, Engberg S, Matthews JT, Dunbar-Jacob J, McCarthy CE et al (2002) Pearl: a mobile robotic assistant for the elderly. AAAI workshop on automation as eldercare 2002:85-91

33. Psomopoulos F, Tsardoulias E, Giokas A, Zielinski C, Prunet V, Trochidis I, Daney D, Serrano M, Courtes L, Arampatzis S, et al (2014) RAPP system architecture. In: IEEE/RSJ international conference on intelligent robots and systems assistance and service robotics in a human environment

34. Reppou S, Karagiannis G (2015) Social inclusion with robots: a RAPP case study using NAO for technology illiterate elderly at Ormylia Foundation. In: Progress in automation, robotics and measuring techniques. Springer, $\mathrm{p} 233-241$

35. Rizzi L, Rosset I, Roriz-Cruz M (2014) Global epidemiology of dementia: Alzheimers and vascular types. Biomed Res Int 2014:e908915

36. Rogers WA, Meyer B, Walker N, Fisk AD (1998) Functional limitations to daily living tasks in the aged: a focus group analysis. Hum Factors J Hum Factors Ergon Soc 40(1):111-125

37. Schulte J, Rosenberg C, Thrun S (1999) Spontaneous, short-term interaction with mobile robots. In: IEEE international conference on robotics and automation, 1999. Proceedings, vol 1. IEEE, Detroit, MI, p 658-663

38. Smith R, Quine S, Anderson J, Black K (2002) Assistive devices: self-reported use by older people in Victoria. Aust Health Rev 25(4):169-177

39. Tenorth M, Beetz M (2009) Knowrob: knowledge processing for autonomous personal robots. In: IEEE/RSJ international conference on intelligent robots and systems, 2009. IROS 2009. IEEE, Detroit, MI, p 4261-4266

40. Tiberio L, Padua L, Pellegrino AR, Aprile I, Cortellessa G, Cesta A (2011) Assessing the tolerance of a telepresence robot in users with mild cognitive impairment. In: Proceedings of HRI 2011 workshop on social robotic telepresence, Lausanne, 6 March 2011, p 23-28

41. Tsardoulias EG, Zieliński C, Kasprzak W, Reppou S, Symeonidis AL, Mitkas PA, Karagiannis G (2015) Merging robotics and AAI ontologies: the RAPP methodology. In: Progress in automation, robotics and measuring techniques. Springer, p 285-297

42. Walker W, Lamere P, Kwok P, Raj B, Singh R, Gouvea E, Wolf P, Woelfel J (2004) Sphinx-4: a flexible open source framework for speech recognition

43. World Health Organization (2012) Dementia: a public health priority. World Health Organization, Mental health, Geneva. http:// www.who.int/mental_health/publications/dementia_report_2012/ en/. Accessed 8 Nov 2015

44. World Health Organization 2015 dementia facts. http://www.who. int/mediacentre/factsheets/fs362/en/. Accessed 15 Nov 2016 
Sofia E. Reppou obtained her MSc Degree from the Psychology Department of Sheffield University and her BSc Degree from Aristotle University of Thessaloniki. She also holds an executive MBA from the University of Macedonia of Thessaloniki. She was engaged for several years in managerial positions at Vodafone Telecommunications and has extended experience in project and human resources management. Her research interests include human-robot interaction, social psychology, education for people at risk of exclusion and e-learning. She is a currently a Research Associate with the Ormylia Foundation working on RAPP (FP7 EU): RApps for Delivering Smart User Empowering Applications.

Emmanouil G. Tsardoulias has obtained his Doctorate and Engineering Diploma from the Department of Electrical and Computer Engineering at Aristotle University of Thessaloniki (AUTH), Greece, in 2013 and 2007, respectively. His working experience includes participating in the "Eudoxus" Project with the collaboration of Ministry of Education and the RAPP Project (FP7-ICT-610947). His research interests are focused in robotics and specifically in autonomous robots (navigation, SLAM and multi-robot exploration/full coverage). In addition, from 2009 he is the Head Manager of the AI Group of the Robotics Team PANDORA, which operates at the School of Electrical and Computer Engineering, Aristotle University of Thessaloniki.

\begin{abstract}
Athanassios M. Kintsakis received his Diploma in Electrical and Computer Engineering from the Aristotle University of Thessaloniki in July 2014. Currently he is pursuing his Doctoral Degree in the same department in the field of High Performance Computing Systems applied in Bioinformatics. His research interests include software engineering, data mining and machine learning in a cloud computing environment. Since November 2014 he is working as a Software Engineer in European Union Research Projects and as a teaching assistant in various department courses.
\end{abstract}

Andreas L. Symeonidis (users.auth.gr/symeonid) is an Assistant Professor with the Department of Electrical and Computer Engineering at the Aristotle University of Thessaloniki and a Faculty Affiliate with the Information Technologies Institute. His research interests include software engineering (SE) processes, knowledge extraction from software repositories, model-driven engineering, software quality and software analytics. Dr. Symeonidis' work has been published in over 80 journal and conference papers. He is co-author of the book "Agent Intelligence through Data Mining" (ISBN 0-387-24352-6), and editor of 5 Springer lecture notes. Dr. Symeonidis is currently Project Coordinator of S-CASE (FP7-ICT-610717), RAPP (FP7-ICT-610947) and Technical Coordinator for the Pandora robotic vehicle projects.

Pericles A. Mitkas received his Diploma of Electrical Engineering from AUTH (1985), an MSc and a PhD in Computer Engineering from Syracuse University, USA, in 1987 and 1990, respectively. He is currently a Faculty Member of the Department of Electrical and Computer Engineering of AUTH and a Faculty Affiliate of the Information Technologies Institute of CERTH, where he directs the Intelligent Systems and Software Engineering Laboratory. His research interests include databases and knowledge bases, data mining, software agents, enviromatics and bioinformatics. Professor Mitkas is a Senior Member of the IEEE Computer Society and has served as Project Coordinator in several US and EU research projects. His work has been published in over 220 papers, book chapters, and conference publications. Currently Professor Mitkas is the elected Rector of AUTH.

Fotis E. Psomopoulos is currently an Academic Fellow at AUTH (Aristotle University of Thessaloniki) and a Research Associate at the Centre for Research and Technology Hellas (CERTH). Since 2005 he has been working on several EU-funded research projects, and has organised several seminar lectures on bioinformatics, as well as two international EGI-affiliated workshops on the use of grid and cloud computing in life sciences. His research interests lay across three major pillars; (a) bioinformatics and the analysis of sequence data, (b) data mining methods, and (c) grid and cloud computing in order to facilitate large-scale approaches.

George T. Karagiannis Electrical and Computer Engineer, $\mathrm{PhD}$. He is a Scientific and Technical Head of the Diagnosis Centre of the Ormylia Foundation. He is Scientific Collaborator of Ormylia Foundation since January 1997. Since 1998, he is involved as coordinator and principal participant in European R\&D as well as industrial projects related to NDT techniques development and application in art objects diagnosis and documentation, biomedical diagnosis, image and signal processing and knowledge management. He is a Reviewer in several scientific journals and evaluator of European Commission for RTD projects. He is a Member of technical and scientific committees of international conferences.

Cezary Zielinski received MSc/Eng Degree in control in 1982, PhD Degree in Control and Robotics in 1988 and DSc (Habilitation) Degree also in Control and Robotics in 1996, all from Warsaw University of Technology (WUT), Faculty of Electronics and Information Technology (FEiTI), Warsaw, Poland. He is a Professor of WUT employed by the Institute of Control and Computation Engineering (IAIS). His research interests focus on robotics in general and especially include: robot programming methods, multi-robot system controllers, robot kinematics, robot force control, visual servo control, utilisation of sensors in robot control, behavioural and hybrid control of robots, mechatronics, and design of digital circuits. He is the Author/Co-author of over 150 conference and journal papers.

Vincent Prunet is a Senior Research Engineer at Inria. He is an experienced manager of ICT ventures and projects, with a strong R\&D and industry background in software engineering, embedded software, telecommunications and security. He joined Inria in 2012 where he is in charge of turning software innovations (Hop.js : a language and software tools to develop applications for robots and IoT using web technologies) into applications and fostering technology transfer. He graduated from Paris' Ecole Normale Suprieure and holds a PhD in Solid State Physics and an MS in Software Engineering (1989).

Jean-Pierre Merlet received the MS Degree in Mathematics from the University of Nantes, Nantes, France, in 1978, the Engineer Title from the National Superior School of Mechanics, Nantes, in 1980, the PhD Degree from Paris VI University, Paris, France, in 1986, and the Research Habilitation Degree from Nice University, Nice, France, in 1993. He is currently a Senior Researcher with Inria Sophia-Antipolis, Mediterranee, France. He has authored or coauthored over 200 conference and journal papers in the field of force control of robots, algebraic geometry, constraint solving, and parallel robots, and a book on the latter subject with two editions in French and two editions in English (Springer-Verlag, 2001 and 2005). 
Miren Iturburu holds a Bachelor's Degree in Chemistry from the University of the Basque Country (Spain). She is Expert in Psychosocial Intervention in Gerontology and holds a Master Degree in Aging at the University of Murcia. Currently she works at the Matia Institute as Researcher in the field of ageing in different projects related to the elderly and disabled people. Within Matia she coordinates and participates in different national and European research projects (ITEA2, AAL, FP6, FP7 and H2020) and has conducted pilot trials in projects such as: RAPP, Soprano, Agnes, Bedmond, Flexidesc, Saleme, Senifood, Canox, DM2 and others.
Alexandros Gkiokas is a Senior Software Developer for Ortelio Ltd., working on robotics software development, EU-funded research projects, and other AI-related software development in deep learning, machine learning and reinforcement learning. He is a final-year PhD Student at the University of Warwick, part of the Intelligent and Adaptive Systems Group, doing research on Imitation Learning, Learning by Example and Knowledge Representation. He has over 10 years of industrial experience, working across disciplines, from ML, NLP to robotics, and lately in cloud computing and virtualisation. He speaks four languages and is a Member of the ACM. 